\title{
The effect of controllable and uncontrollable neonatal preshocks on adult escape/avoidance behavior in the guinea pig (Cavia porcellus)
}

\author{
FRED FENTON, ANNE CALOF, and RICHARD KATZEV \\ Reed College, Portland, Oregon 97202
}

\begin{abstract}
Neonatal guinea pigs were given escapable, inescapable, or no shock and were later tested as adults on a signaled escape/avoidance task. During the neonatal period, the animals that could escape shock learned to do so quickly and steadily increased their overall level of activity, while those that could not, displayed a consistent decline in activity. Furthermore, during adult escape/avoidance sessions, guinea pigs, that could control neonatal shock were superior to those that lacked such control. These findings extend the generality of the interference effect to the guinea pig and highlight the influence of early control of aversive events on this phenomenon.
\end{abstract}

The occurrence of considerable intraspecies variability is one of the most serious problems besetting current laboratory investigations of the interference (or "learned helplessness") effect in animals. For example, exposure to uncontrollable aversive stimuli does not always retard the subsequent acquisition of instrumental escape/avoidance behavior. Indeed, in their original experiments with dogs, Maier and Seligman (1976) reported that $33 \%$ of those animals given inescapable preshocks subsequently learned quite normally in the shuttlebox. Furthermore, $6 \%$ of the dogs failed to learn even though they had never been previously exposed to shock. In trying to account for this variability, Maier and Seligman suggested that it is essential to consider the animal's prior history. For example, those that have had considerable early experience controlling aversive stimuli may be immune to the effects of inescapable aversive events in adulthood, while those that have had no such experience or have had extensive prior exposure to uncontrollable aversive events may be particularly retarded when exposed to adult trauma.

In an early test of these notions, Seligman and Groves (1970) reported that laboratory-reared dogs with known life histories involving minimal exposure to stress displayed greater deficits following exposure to uncontrollable shocks than supplier-provided dogs with unknown life histories. In a more recent study, Hannum, Rosselini, and Seligman (1976) reported that inescapable shock given to weanling rats led to large deficits in adult learning to escape shock on a leverpress (FR 3) task. They also reported that exper-

Requests for reprints should be sent to Richard Katzev, Department of Psychology, Reed College, Portland, Oregon 97202. The authors thank Sheryl Schumacher for conducting pilot studies on avoidance learning in the guinea pig. ience with escapable shock while a weanling protected their adult rats in this situation against the debilitating effects normally produced by prior exposure to inescapable shock.

Unfortunately, Hannum et al. (1976) fail to report the number of rats that were not "helpless" in each condition, and thus it is impossible to assess the degree to which adult variability was reduced by these early experiences. However, it is clear from their published figures that some of the inescapably shocked rats performed escape responses during the adult testing sessions. It is equally clear that some of the rats in both the controllable shock and nonshock conditions were unable to do so. Assuming that their postweaning histories were comparable, it is possible that this variability could have been produced by differential preweaning experiences. Since Hannum et al. (1976) could not introduce controllable shock until their rats had been weaned, the influence of such preweaning experiences cannot be ruled out in accounting for behavioral differences in this species.

The following study was designed to explore this possibility by investigating the interference effect in the precocial guinea pig. The guinea pig (Cavia porcellus) has been bred for generations to yield characteristics suitable for biological research. However, many of these same characteristics make it a particularly useful subject for behavioral investigations, especially those concerned with the effects of early experience. Unlike the rat, the guinea pig is a precocial species; it can see, run, and eat solid food within minutes after birth (Johnson, Lyle, Edwards, \& Penny, 1975). Its average birthweight is $100 \mathrm{~g}$ as opposed to $6 \mathrm{~g}$ in rats, and complete weaning usually occurs by 10 days. Since the newborn guinea pig is relatively mature and mobile, it can adapt much earlier than the rat to experimentally induced trauma. Thus, it is possible to undertake controlled experimental 
manipulations of the guinea pig within hours after birth.

\section{METHOD}

\section{Subjects}

Twenty-one experimentally naive American short-hair guinea pigs (Cavia porcellus) were employed in this study. They consisted of 13 males and 8 females selected from the offspring of nine breeders purchased from a local supplier 1 month prior to parturition. Following the neonatal treatments, the animals were separated from their mothers at 21 days and left undisturbed in individual housing enclosures until the start of adult testing at 90 days. Throughout the experiment, the animals were maintained in a constant 14-h-light/10-h-dark cycle. Purina Guinea Pig Chow, which contains a vitamin $C$ supplement and water, supplemented with Tang, were available on an ad-lib basis.

\section{Apparatus}

Phase 1. All sessions during the neonatal period were conducted in a two-way shuttlebox (Lehigh Valley Electronics), incorporating a tilting floor with metal grid floor-bars spaced every $5 \mathrm{~mm}$ to allow the neonates to move about without difficulty. A response was recorded each time the animal crossed from one side of the box to the other. The initial neonatal treatments were conducted in a specially designed Plexiglas enclosure placed over the grid floor. This enclosure, in the form of an inverted trough with trapezoidal sides, was $8.5 \mathrm{~cm}$ wide and $9 \mathrm{~cm}$ high, and had a roof length of $31 \mathrm{~cm}$ and a larger base extending the full length of the $45-\mathrm{cm}$ grid floor. The enclosure prevented false shuttling responses due to excessive running or jumping, and it minimized responses incompatible with shuttling

Illumination was provided by two $15-\mathrm{W}$ bulbs located in the ceiling of the sound-attenuating chamber. White masking noise of $66 \mathrm{~dB}$ was presented at all times through a speaker mounted on the roof of the shuttlebox. A constant current shock of $1 \mathrm{~mA}$ was delivered to the grid floor by BRS Foringer shock generating and scrambling units. The recording of all responses, as well as the onset and offset of shock, were also controlled by BRS Foringer solid-state electronic equipment. A Roytron eight-channel papertape punch recorded the latency of each response in the escapable shock condition so that an identical sequence and patterning of shocks could be delivered later to animals in the yoked, inescapable shock condition.

Phase 2. Adult escape/avoidance conditioning took place in a one-way jump box, manufactured by Lafayette Instrument Co. The upper platform ledge was closed, providing a lower grid area measuring $23 \times 20 \times 20.5 \mathrm{~cm}$ where test trials were conducted. The grid floor consisted of 15 stainless steel bars placed $1.5 \mathrm{~cm}$ apart. The manipulandum was a $7 \times 15 \mathrm{~cm}$ metal plate mounted on a hinge in the center of one of the Plexiglas sides. A response was recorded whenever this plate was depressed laterally; the animals usually performed this response by pressing the plate with their noses or front paws. Holding the plate had no effect on either the onset or the of fset of a trial.

The chamber was housed within a larger, sound-attenuating enclosure. Illumination was provided by one $15-\mathrm{W}$ bulb located on the wall of this enclosure and, during all experimental sessions, a masking 75-dB white noise was delivered through a speaker mounted on the roof of the one-way box. The conditioned stimulus (CS) consisted of a $90-\mathrm{dB}$ tone delivered through the same speaker. The unconditioned stimulus (US) was a $1-\mathrm{mA}$ scrambled shock delivered to the grid floor by a Lafayette Master Shocker on $100 \%$ duty cycle. The presentation of stimuli and recording of responses were automated by BRS-Foringer programming equipment.

\section{Procedure}

Phase 1. Daily observations were made on the mothers so that the initial sessions could be administered within $24 \mathrm{~h}$ after birth.
After they were born, the neonates were randomly assigned to the escapable shock, yoked inescapable shock, or the no-shock control group, each consisting of seven animals. All animals were given a total of eight sessions, with an intersession interval of $48 \mathrm{~h}$. Each animal received a 5 -min habituation period before the start of the first session. Because of the growth of the guinea pigs, the Plexiglas enclosure was used only through Session 5.

In the escape condition, the animals received eight sessions of escape training, consisting of 50 trials in which shock was presented on a VI 60-sec intertrial interval, with a range of 30-120 sec. The maximum duration of shock on all sessions was $10 \mathrm{sec}$. During the first two sessions, any crossing that occurred in less than $10 \mathrm{sec}$ terminated shock and was recorded as an escape response. Beginning with Session 3, in order to insure that the animals in the yoked condition would receive shock of sufficient duration to minimize adventitious escape responses, a minimum of 2 sec of shock was given; responses less than $2 \mathrm{sec}$ were recorded, but they did not terminate shock and were not counted as escape responses. During Sessions 4-8, the minimum duration of shock was $3 \mathrm{sec}$.

In the yoked condition, animals were given the same duration of shock in identical temporal order as their escape partners; however, their responses had no effect on the termination of shock. Animals in the nonshock control condition were simply placed in the chamber for $55 \mathrm{~min}$ on the first session and for $50 \mathrm{~min}$ during the remaining seven sessions. All shuttling responses in these latter two groups were recorded. The animals were weighed before each session, and handling procedures were standardized by transporting the animals from their home cage to the shuttlebox in the weighing chamber.

Phase 2. When the guinea pigs reached 90 days of age, they were given escape/avoidance conditioning trials. Each trial began with the onset of the tone CS. The US was scheduled to be delivered $10 \mathrm{sec}$ later. If the animal pressed the plate within $10 \mathrm{sec}$, the CS terminated immediately and shock was avoided. If no such response occurred within $10 \mathrm{sec}$, shock was delivered and both the CS and the US remained on until the pig pressed the plate. If no response occurred after $15 \mathrm{sec}$, the trial terminated automatically.

The animals were habituated to the one-way chamber for $15 \mathrm{~min}$ prior to the start of the first session. Four escape/avoidance conditioning sessions were given, each consisting of 50 trials presented on a VI $60-\mathrm{sec}$ intertrial interval with a range of $20-220 \mathrm{sec}$. On the fourth session, training was continued until each animal met a criterion of 8 out of 10 consecutive avoidance responses or until 50 trials had been given. The animals were run at the same time of day with sessions separated by a 48-h interval. Handling procedures were standardized as in Phase 1 and the animals were returned to their home enclosures immediately after the completion of each session.

\section{RESULTS}

\section{Phase 1}

Within $24 \mathrm{~h}$ after birth, most of the guinea pigs in the escape group were able to escape shock on almost all 50 trials. During the initial session, the mean number of escape responses for animals in this group was 46 , with a range of 38-50, a level of performance maintained throughout the course of the neonatal sessions (overall mean $=45.8$; range 34.8 49.9).

Figure 1 presents the overall activity level (all intertrial and escape responses combined) for the three groups over the sessions of Phase 1. Animals in the escape condition displayed a consistent rise in activity during the initial sessions and then leveled off at a relatively high rate during the remaining sessions. In contrast, yoked animals displayed a consistent de- 


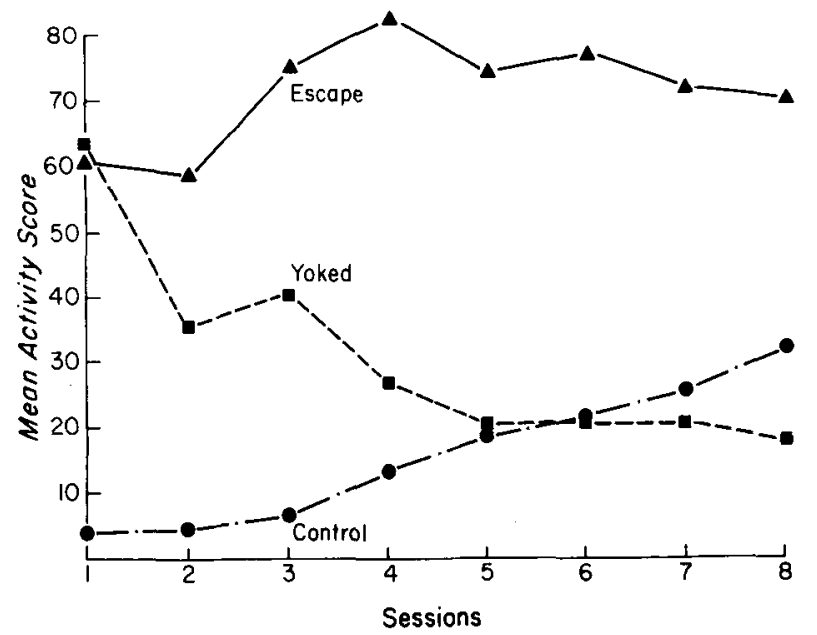

Figure 1. Mean activity score (escape and intertrial responses combined) for each group during the eight neonatal sessions.

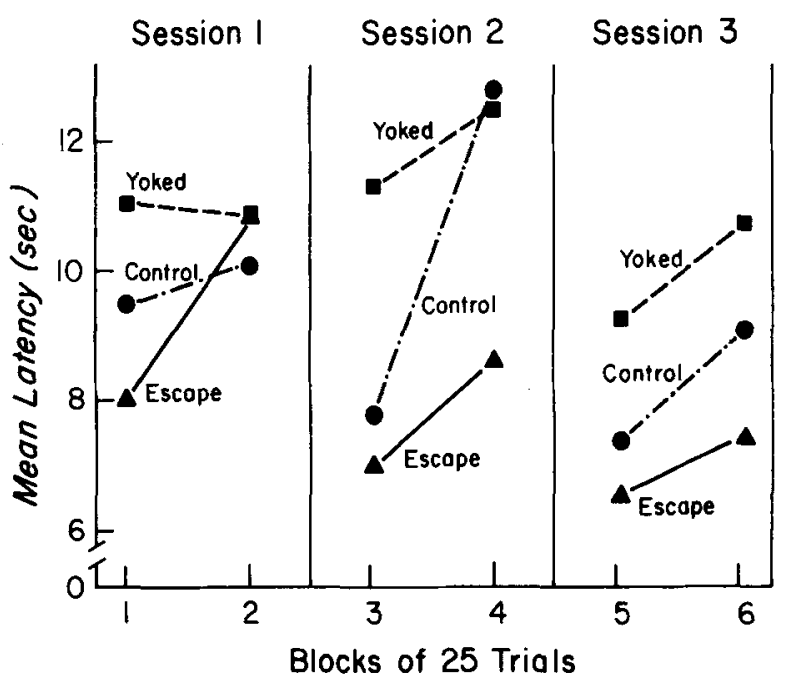

Figure 2. Mean latency to respond (seconds) for each group in blocks of 25 trials.

crease in activity during the early sessions and then stabilized at a relatively low level. Animals in the control group made few crossings initially, although they responded with increasing frequency as the sessions continued. An analysis of total activity scores revealed that the escape group differed significantly from both the yoked and control group throughout the neonatal sessions $(T=0, p<.01 ; U=0, p=0$, respectively), as well as on the last neonatal session $(\mathrm{T}=0, \mathrm{p}<.01 ; \mathrm{U}=7, \mathrm{p}<.01$, respectively $) .^{1}$ The yoked and control groups did not differ over the course of the eight sessions, although on the last neonatal session yoked animals were significantly $(\mathrm{U}=$ $11, \mathrm{p}<.05$ ) less active than control animals.

\section{Phase 2}

In agreement with the results of other investigators (Sansone \& Bovet, 1970; Webster \& Rabedeau, 1964), the adult guinea pigs in this study displayed a marked within-session decrement in responding. Analysis of the overall latency to respond during the first and second half of a session revealed that the animals responded significantly $(T=1, p<.01)$ faster during the first 25 trials than they did during the second. In spite of this within-session decrement, Figure 2, which plots the mean latency to respond in 25-trial blocks for each group during Phase 2 , reveals that animals in the escape group consistently responded more quickly than animals in other groups. Due to the intersession decrement, there were no significant differences between any of the groups during the second 25 trials. However, during the first 25 trials, the escape group responded significantly more rapidly than the animals in the yoked group. This was true when the latency measure was combined across all three test sessions $(T=2, p<.025)$ as well as from the outset of adult testing during the first 25 trials of Session $1(T=1, p<.025)$.

A similar pattern emerged in the analysis of avoidance responses, which are plotted in Figure 3 (Sessions 1-3). Animals in the escape group avoided at a steadily increasing rate and were clearly superior to those in the yoked condition. Control animals also avoided with increasing frequency so that by the end of the 3rd day they were responding at a level which was indistinguishable from that of the escape group. An analysis of the total number of avoidance responses during Days 1-3 of testing reveals that escape and control groups did not differ on this measure, although they each performed significantly more avoidance responses than animals in the yoked group ( $\mathrm{T}=2, \mathrm{p}<.025 ; \mathrm{U}=10, \mathrm{p}<.036$, respectively).

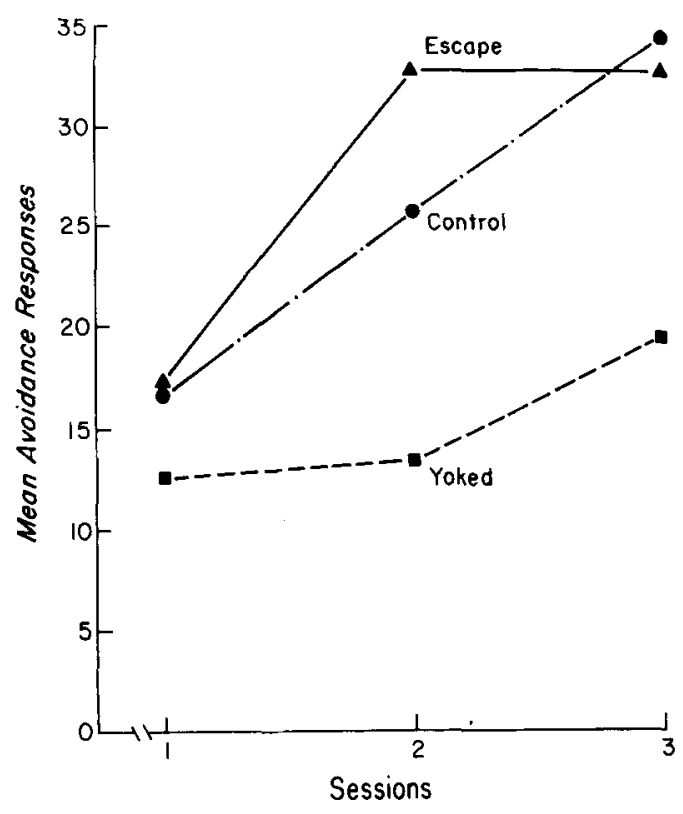

Figure 3. Mean number of avoidance responses for each group during the adult test sessions. 
These trends continued on Day 4 when the animals were tested on a criterion of 8 out of 10 avoidance responses. Most of the animals in the escape and control conditions ( $86 \%$ and $71 \%$, respectively) met this criterion by the end of the 50-trial session while only $43 \%$ of the yoked animals did. While the animals in the escape group required fewer trials to reach the criterion than those in the control group, the diffcrence between these two groups was not significant. However, both escape and control animals took significantly fewer trials to reach the avoidance criterion than animals in the yoked group $(T=0, p<.01$; $\mathrm{U}=8, \mathrm{p}<.01$, respectively).

\section{DISCUSSION}

The results of this experiment indicate that guinea pigs that could control neonatal shock were superior to those that lacked such control during adult escape/ avoidance conditioning trials. This was reflected in measures of response latency, frequency of avoidance responses, and trials to the avoidance criterion. Furthermore, these effects not only appeared during the initial block of trials, but persisted throughout the course of the 4 days of adult testing.

These outcomes corroborate the findings of Hannum et al. (1976), who observed a comparable interference effect in a developmental study of weanling rats. In addition, this study extends their findings in several respects. It widens the generality of the interference effect to the guinea pig and, in so doing, demonstrates that it is a promising species for future research on this phenomenon. In addition, by taking advantage of the guinea pig's early maturity, it makes it possible to extend the developmental analysis of this effect to the preweaning period.

Further, the results of the current study make it clear that an organism's early history plays a crucial role in governing the interference effect. As such, it is consistent with recent research which has highlighted the importance of early control of environmental events in governing adult behavior. For example, Joffee, Rawson, and Mulick (1973) have reported that rats reared from birth in an environment where they could control the occurrence of food, water and lighting were much less emotional, as judged by activity and defecation scores in an open-field test, than rats reared in an environment where these same events were uncontrollable. Other research (Wight \& Katzev, 1977) has demonstrated that these differential developmental contingencies also influence adult learning by showing that prolonged exposure to uncontrollable positive reinforcers impairs the ability of adult rats to escape and avoid shock in a shuttlebox. In short, while the effects of early experience have long been recognized, it is only recently that the dimension of control over these experiences has been considered to be equally important. Indeed, as the yoked-control design of this experiment demonstrates, the same experience can have entirely different effects depending on whether it is controllable or uncontrollable.

The present study sought to carefully regulate such early experiences in order to minimize the variability reported in previous studies of the interference effect. Thus, all living conditions and experimental manipulations were constantly monitored beginning from 1 month prior to the birth of the guinea pigs, through the pre- and postweaning periods, to the completion of adult testing. In spite of this, there was still a considerable amount of variability within each of the treatment conditions. For example, two of the animals in the yoked, inescapable shock condition escaped quickly and learned to avoid at a relatively high rate. In fact, $43 \%$ of the yoked animals reached the avoidance criterion in spite of the fact that they had previously been exposed to inescapable shock. Conversely, one of the animals in the escape condition had lengthy escape latencies and failed to perform a single avoidance response during the 200 test trials. In addition, two of the animals in the control group responded quite slowly and failed to meet the avoidance criterion even though they had never been previously exposed to shock.

It is possible that genetic differences may account for a portion of this variability. In rats, the interference effect is known to be influenced by genetic factors (Shurman \& Katzev, 1975), with some strains of rats seriously impaired by uncontrollable shock, while others are not. Alternatively, this variability might be a function of prenatal experiences, although an effort was made to carefully control living conditions prior to the birth of the guinea pigs. Regardless of their relative contribution, future research on this phenonemon should attempt to gain control of each of these factors by employing inbred strains of animals.

Finally, it is important to point out that the interference effect observed in the current study did not involve the profound disturbances in responding described in the original "learned helplessness" studies. There was no evidence in this study to indicate that inescapably shocked guinea pigs "gave up" or that their behavior resulted from an inability to perform the plate-press response. In fact, all but one of the yoked animals escaped at a high level, independently of whether they learned to avoid. Rather, the behavior of these guinea pigs might better be described as a performance deficit, which was reflected in a slower latency to respond and a reduced frequency of avoiding relative to animals given prior exposure to escapable shock.

\section{REFERENCES}

Hannum, R., Roseli.ini, R., \& Seligman, M. Learned helplessness in the rat: Retention and immunization. Developmental Psychology, 1976, 12, 449-454. 
Joffe, J., Rawson, R., \& Mulick, J. Control of their environment reduces emotionality in rats. Science, 1973, 130, 1383-1384.

Johnson, K., Lyle, J., Edwards, M., \& Penny, R. Problems in behavioural research with the guinea pig: A selective review. Animal Behaviour, 1975, 23, 632-639.

Maier, S., \& Seligman, M. Learned helplessness: Theory and evidence. Journal of Experimental Psychology: General, 1976, 104, 3-46.

Sansone, M., \& Bovet, D. Avoidance learning by guinea pigs. Quarterly Journal of Experimental Psychology, 1970, 22, $458-461$.

Seligman, N., \& Groves, D. Nontransient learned helplessness. Psychonomic Science, 1970, 19, 191-192.

Shurman, A., \& Katzev, R. Escape/avoidance responding in rats depends on strain and number of inescapable preshocks. Journal of Comparative and Physiological Psychology, 1975 , 88, 548-553.
Webster, C., \& Rabedeau, R. The effect of intersession interval in shuttlebox conditioning of the guinea-pig. Psychonomic Science, 1964, 1, 73-74.

Wight, M., \& KatZEv, R. Noncontingent positive reinforcers retard later escape/avoidance learning in rats. Bulletin of the Psychonomic Society, 1977, 9, 319-321.

\section{NOTES}

1. All paired comparisons between the escape and yoked group employed the Wilcoxon matched pairs signed-ranks test $(\mathrm{T})$, while other paired comparisons used the Mann-Whitney $U$ test. All p values are for one-tail tests.

\footnotetext{
(Received for publication May 19, 1978; revision accepted October 24,1978 .)
} 